\title{
Pattern motion selectivity in population responses of area 18
}

\author{
Kerstin E. Schmidt, ${ }^{1}$ Miguel Castelo-Branco, ${ }^{2}$ Rainer Goebel, ${ }^{3}$ Bertram R. Payne, ${ }^{4}$ Stephen G. Lomber ${ }^{5}$ and \\ Ralf A. W. Galuske ${ }^{1,6}$ \\ ${ }^{1}$ Max-Planck-Institute for Brain Research, Deutschordenstraße 46, 60528 Frankfurt, Germany \\ ${ }^{2}$ IBILI, Visual Neuroscience Laboratory, University of Coimbra, Portugal \\ ${ }^{3}$ Department of Cognitive Neuroscience, Faculty of Psychology, Maastricht University, The Netherlands \\ ${ }^{4}$ Anatomy \& Neurobiology, Boston University School of Medicine, USA \\ ${ }^{5}$ Centre for Brain and Mind, Roberts Research Institute, University of Western Ontario, Canada \\ ${ }^{6}$ Department of Biology, Technical University of Darmstadt, Germany
}

Keywords: cat, optical imaging, second-order motion, visual cortex

\begin{abstract}
It is commonly believed that the complexity of visual stimuli represented by individual neurons increases towards higher cortical areas. However, even in early visual areas an individual neuron's response is influenced by stimuli presented outside its classical receptive field. Thus, it has been proven difficult to characterize the coding of complex stimuli at the level of single neurons. We therefore investigated population responses using optical imaging in cat area 18 to complex stimuli, plaids. Plaid stimuli are composed of two superimposed gratings moving in different directions. They may be perceived as either two separate surfaces or as a global pattern moving in intermediate direction to the components' direction of motion. We found that in addition to activity maps representing the individual components' motion, plaid stimuli produced activity distributions matching the predictions from a patternmotion model in central area 18. Thereby, relative component- and pattern-like modulations followed the degree of psychophysical pattern bias in the stimulus. Thus, our results strongly indicate that area 18 exhibits a substantial response to pattern-motion signals at the population level suggesting the presence of intrinsic or extrinsic mechanisms that allow for integration of motion responses from far outside the classical receptive field.
\end{abstract}

\section{Introduction}

Neurons in primary visual cortex are selective for linear motion. However, their receptive fields form small apertures to the visual world in which the true direction of motion of a line segment is underspecified (Marr \& Ullman, 1981; Adelson \& Movshon, 1982). For example, motion parallel to a line's orientation is invisible to a local motion detector as the physical stimulus does not change over time. A family of different directions of motion will be consistent with what is visible within the aperture (Wallach, 1935). Two-dimensional patterns consisting of two superimposed non-parallel gratings (plaids) can be perceived as either two independent gratings sliding on top of each other or as one coherent pattern sliding in an intermediate direction. The direction of pattern motion is thought to be computed in a two-stage hierarchical process (Movshon et al., 1985). In the first stage, 1D components are analysed by orientation- and directionselective mechanisms that evaluate the motion of local contours (component motion). In the second stage, the outputs of the first stage are integrated to construct the true direction of pattern motion.

For both types of motion specialized neurons have been identified. Electrophysiological studies indicate that the majority of neurons in early visual areas of cats and monkeys respond to component-like

Correspondence: Dr Kerstin E. Schmidt, as above.

E-mail: schmidt@mpih-frankfurt.mpg.de

Received 24 October 2005, revised 5 July 2006, accepted 14 August 2006 motion (Movshon et al., 1985; Gizzi et al., 1990; Castelo-Branco et al., 2000; Geisler et al., 2001; Li et al., 2001; Guo et al., 2004). In general, pattern-selective neurons are less frequent and mainly found in higher visual areas with larger receptive fields (Albright, 1984; Movshon et al., 1985; Rodman \& Albright, 1989; Gizzi et al., 1990; Scannell et al., 1996; Gegenfurtner et al., 1997; Castelo-Branco et al., 2000; Li et al., 2001; see also Merabet et al., 1998) but hardly ever in primary visual cortex (Guo et al., 2004).

Nevertheless, it was noted that pattern selectivity can be increased by wakefulness (Pack et al., 2001; Guo et al., 2004) or by stimulus choice (Movshon et al., 1985; Rodman \& Albright, 1989; Stoner \& Albright, 1992; Li et al., 2001). Thus, pattern and component selective neurons do not form completely separated populations implying that a strict two-stage model is questionable.

For example, varying the contrast of the intersections such that the pattern does or does not conform to the rules of transparency biases the percept towards either single component or coherent pattern motion (Stoner et al., 1990; Stoner \& Albright, 1996) and modifies the degree of pattern selectivity in MT (Stoner \& Albright, 1992). In cat area 18 , individual neurons were rarely found to respond pattern-like, even to non-transparent plaid stimuli, which strongly bias pattern perception (Castelo-Branco et al., 2000). Given this sparseness of pattern responses and mutual interactions among large groups of neurons we decided to investigate population responses. To this end, we presented plaid stimuli of different pattern-bias and monitored 
population responses with optical imaging of intrinsic signals. We compared maps evoked by plaid stimuli in area 18 with maps evoked by orientated gratings moving into the direction of the plaids' components or into the pattern direction of motion. Intrinsic activity related to both pattern and component motion could be observed.

\section{Materials and methods}

\section{Animal preparation}

The study was approved by the ethical committee for animal experimentation of the Government of Hessen. All experimental procedures were performed in accordance with the Society for Neuroscience and the German laws for animal protection. Optical imaging of intrinsic signals was performed in area 18 (34 data sets) of 12 adult ( $>1$ years) cats. Data sets from five of the cats, different from the ones reported here, have been published as part of another study (Galuske et al., 2002). Anaesthesia was initiated by intramuscular injection of ketamine $(10 \mathrm{mg} / \mathrm{kg}$; Ketamin, CEVA Tiergesundheit $\mathrm{GmbH}$, Düsseldorf, Germany) and xylazin (1 mg/kg, Rompun, Bayer Vital, Leverkusen, Germany) and was maintained after tracheotomy by artificial ventilation with a mixture of $\mathrm{N}_{2} \mathrm{O}(70 \%), \mathrm{O}_{2}(30 \%)$ and halothane (1.2\%, Halothan, Eurim-Pharm Arzneimittel GmbH, Piding, Germany) supplemented with intravenous application of a muscle relaxant (pancuronium, $0.25 \mathrm{mg} / \mathrm{kg} / \mathrm{h}$, Pancuronium, CuraMED Pharma $\mathrm{GmbH}$, Karlsruhe, Germany) to prevent eye movements. The depth of anesthesia was evaluated by continuously monitoring the electrocardiogram and the expired $\mathrm{CO}_{2}$ level. Imaging chambers were positioned over the midline at AP0 with the recorded surface in the anterior chamber part.

\section{Visual stimulation}

Visual stimuli were presented on a 21 -inch computer screen (Hitachi, CM815ET, refresh rate, $100 \mathrm{~Hz} ; 600 \times 800$ pixels resolution) at a distance of $57 \mathrm{~cm}$. Maps of orientation preference were obtained in the central visual field representation of area 18 using whole-field squarewave gratings of four orientations $(0.1 \mathrm{cyc} / \mathrm{deg}$, duty cycle 0.5$)$ drifting in the two orthogonal directions at $16 \mathrm{deg} / \mathrm{s}$. After having obtained good quality signals, a retinotopic mapping procedure was applied to align the centre of the monitor to the recorded cortical region and thus ensuring optimal stimulation. To this end, the monitor was divided into 12 squares of $10 \mathrm{~cm} \times 10 \mathrm{~cm}\left(\sim 10^{\circ} \times 10^{\circ}\right.$ visual field angle) in which vertical and horizontal square-wave gratings drifted back and fourth in the orthogonal directions in random order. Differential orientation maps were calculated by dividing images obtained with vertical and horizontal stimulation of the same square. Subsequently, the monitor was centred on the position of the square that evoked the strongest activation in the recorded area.

Plaid stimuli were constructed such that a perceptual bias towards pattern motion was based on violations of the rules of physical and perceptual transparency (human Stoner et al., 1990; Stoner \& Albright, 1996; monkey: Thiele \& Stoner, 2003). In this way we could generate two very simple categorical sets of plaid stimuli pattern-biased and non-pattern-biased. Non-pattern-biased stimuli were constructed either by optimizing stimulus transparency or by emphasizing depth-ordering rules that allow for stimulus manipulations that do not depend on grating intersections. To construct the plaid stimuli, square-wave gratings were superimposed in a circular display subtending $24^{\circ}$ of visual angle. The velocity of the pattern movement was $8 \%$, the angle between the directions of the two gratings $150^{\circ}$. The duty cycle was maintained constant at 0.2 , except
TABLE 1. Luminance values of plaid stimuli

\begin{tabular}{llllll}
\hline & \multicolumn{5}{c}{ Luminance of plaid stimuli $\left(\mathrm{cd} / \mathrm{m}^{2}\right)$} \\
\cline { 2 - 6 } & \multicolumn{5}{c}{ Intersection } \\
Stimulus & Component 1 & Component 2 & Plaid & Background Bias \\
\hline PB 1 & 10.08 & 10.08 & 0.162 & 5.25 & Pattern \\
PB 2 & 10.08 & 10.08 & 53.71 & 5.25 & Pattern \\
NPB 1 & 10.08 & 3.33 & 9.5 & 5.25 & Non-pattern \\
NPB 2 & 10.08 & 10.08 & 9.5 & 5.25 & Non-pattern \\
\hline
\end{tabular}

for one control experiment in which the duty cycle was varied $(0.2$, $0.4,0.6$ and 0.8). Two non-pattern-biased and two pattern-biased plaids were constructed (Table 1): One plaid stimulus (non-patternbiased 1, NPB 1) was composed of two gratings of different luminance and in a depth-ordered configuration, which introduces the strongest component bias, both from the point of view of stimulus construction and perception (Stoner et al., 1990). A second plaid stimulus was composed of two gratings of the same luminance as the darker grating in NPB 1 and low contrast at the intersection (nonpattern-biased 2, NPB 2). The two remaining plaid stimuli were strongly pattern-biased because they were composed of the symmetric (iso-luminant) gratings as NPB 2 but strong contrast at the intersections caused by either very low ('black' intersection, pattern-biased 1, PB 1) or very high luminance ('white', pattern-biased 2, PB 2).

In the first experimental protocol we have used non-pattern-biased plaids with depth-ordering (NPB 1) and pattern-biased (PB 1) plaids with black intersections, which induce a very strong perceptual bias. Both plaid stimuli were moved randomly in eight different directions (16 stimuli) in order to activate all possible orientation and direction domains. The compound plaid maps were compared to those obtained by stimulating with moving square-wave single gratings. Data obtained with this protocol are from seven data sets in five cats. The second experimental stimulus protocol consisted of seven different stimulus types and is depicted in Fig. 3 (drawings below and above the activity maps). Four different plaid stimuli with upward direction of pattern motion $\left(90^{\circ}\right)$, two moving grating stimuli of the same orientation, direction of motion and speed of the plaid components, and one grating stimulus representing the orientation, direction of motion and speed of the pattern direction of the plaid stimulus were presented randomly. Data obtained with this protocol are from 33 data sets in 11 cats. A third control stimulus PB 1 with variation of the duty cycle $(0.2,0.4,0.6$ and 0.8$)$ was run in one additional cat.

\section{Eye movements}

We tried to obtain a measure of whether PB and NPB plaid stimuli induce a perceptual pattern or non-pattern bias also in cats. Therefore, we attempted to establish a relationship between the cat's eye movements and the direction of pattern or component motion of the plaid stimuli in awake cats. In this second series of experiments, eye movements were measured with an infrared eye tracker (Ober2, Permobil Meditech, Timra, Sweden) while the cat's head was fixated in a stereotaxic frame. Cats were trained to fixate in the centre of the presented stimulus (over a period of about two months, they were allowed to become accustomed to the frame and head fixation by means of a cat bag, and rewards with milk and special food which was not part of their normal diet). The distribution of saccades during presentation of the pattern-biased stimulus PB 1 (left) and the nonpattern biased stimulus NPB 1 (right) was measured in two restrained cats. It may be difficult to establish a clear relation between eye 

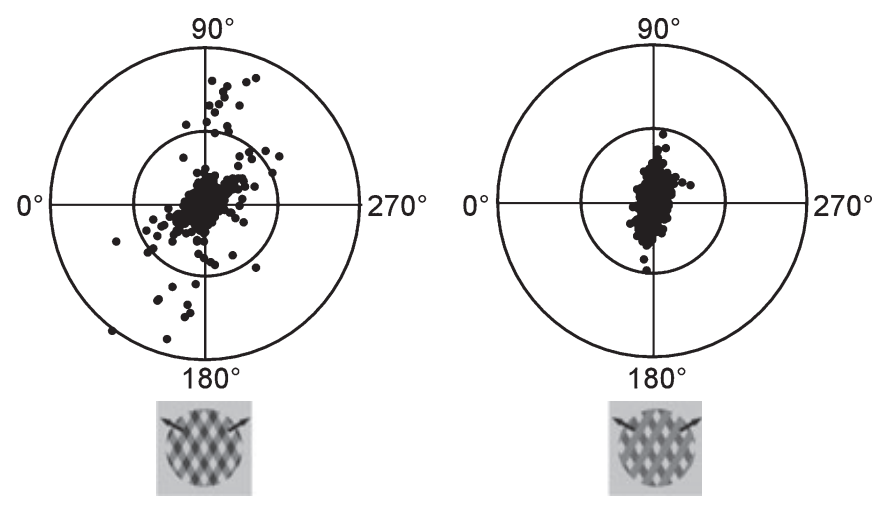

FIG. 1. Eye movements during plaid stimulation. Distribution of saccadic eye movements during presentation of the pattern-biased stimulus PB 1 (left) and the non-pattern biased stimulus NPB 1 (right) in two cats. The inner circle corresponds roughly to $5^{\circ}$ and the outer circle to $10^{\circ}$ of visual field angle. The pattern direction of motion of the plaids stimuli corresponds to vertically upwards. Cats were trained to fixate (the fixation point is localized in the centre of the cross). Nevertheless, eye movements to the PB plaid are more widespread than to the NPB plaid.

movements and pattern/component perception, particularly when the animals are instructed to fixate. Although, we could not identify a preference direction of gaze during plaid stimulation we see a condition dependent change in fixation stability (Fig. 1). The plots of eye movements during the presentation of NPB 1 show much less spread points, which suggests that it is easier to keep fixation under non-pattern biased conditions. This could be the case if the animal perceives two surfaces.

\section{Optical data acquisition}

For optical imaging, chambers were filled with silicone oil and sealed with a glass plate. During recording periods, the level of halothane was lowered to $0.8 \%$. Signals were recorded with a cooled CCD camera system (Ora, 2001, Optical Imaging Europe, Munich) under illumination with light of a wavelength of $560 \mathrm{~nm}$ for surface images and $620 \mathrm{~nm}$ for functional imaging. The camera was fitted with a macroscope providing a $3.6 \times 4.8 \mathrm{~mm}$ field of view (40 pixels $/ \mathrm{m}^{2}$ cortex). For functional imaging, the camera was focused $600 \mu \mathrm{m}$ below the pial surface. Stimuli for the grating protocols were presented for $5 \mathrm{~s}$ (interstimulus interval $10 \mathrm{~s}$ ) and stimuli for the plaid protocols were presented for $7.2 \mathrm{~s}$ (interstimulus interval $15 \mathrm{~s}$ ). Camera frames of $600 \mathrm{~ms}$ duration were collected during the entire stimulus presentation. Usually, individual stimuli were repeated 24 times.

\section{Optical data analysis}

All images were filtered using box-car filters (high-cutoff 50 pixels and low-cutoff 5 pixels). Single condition images as well as orientation preference maps evoked by gratings or plaids (first protocol) were calculated by normalization to the sum of all different stimulus conditions within a stimulus set of eight gratings, nonpattern-biased or pattern-biased plaids ('cocktail blank').

For the construction of 'angle maps', activity maps obtained with moving gratings or plaids of opposite direction but same orientation were lumped together. Preferred angles of orientation were computed by pixelwise vectorial addition of the activity in the single-condition maps. For display, the orientation preference was colour-coded. In both grating and plaid angle maps the colour code refers to the pattern direction of motion. Orientation response profiles (Fig. 2B) were computed by superimposing horizontal-vertical orientation difference maps on angle maps (see also Basole et al., 2003). Orientation tuning in grating and plaid maps was compared by pixelwise subtraction of preference angles.

In the second protocol containing seven stimuli, plaid maps were calculated as differential maps by dividing maps obtained with plaid stimuli by the sum of maps obtained by the two component stimuli ['component divisor' (CD), which isolates the pattern response of the map] or by dividing the respective plaid condition by the map obtained with the grating moving in the pattern direction ['pattern divisor' (PD), which isolates the component response of the map]. The differential activity maps were superimposed on the angle maps obtained with orientated gratings and plaid specific activation was coded by brightness of the colour in the underlying angle map. For quantification, a response profile of the amount of plaid specific activation per pixel after normalization (differential plaid activity) with the models was plotted against the direction preference obtained during stimulation with orientated gratings and categorized into 18 classes of $10^{\circ}$-increments. Thereby, opposite directions in the grating maps were lumped together. To compare different data sets, levels of relative activity were normalized and expressed as per cent of the mean relative activity per plaid map.

To obtain correlation measures, component and pattern correlation coefficients (Spearman rank) were obtained between the data and component-model maps (obtained by summing the maps of the two component gratings) and the pattern-model map (obtained by the grating moving in the pattern direction), respectively.

Mean pattern and component indices for the four different plaid stimuli were computed by averaging the respective Spearman rank correlation coefficients obtained in all single data sets. In our study, different data sets were obtained from two different recording positions within area 18 and different hemispheres within 12 different cats. As within the same cat, pattern and component indices varied for different recording sites, we decided to treat different data sets of one cat as separate measurements.

The Fourier energy of all four plaid stimuli was computed. The respective Fourier amplitude spectra are illustrated in Fig. 7A. Energy in the centre bin was set to 0 because it contains most of the Fourier energy but no information about the direction. In order to create a prediction based on Fourier energy the spectra were analysed with respect to the relative power in the two component directions and the pattern direction of motion. To this end, Fourier energy in $10^{\circ}$ compartments of the stimulus was computed and expressed as power in the respective directions relative to overall stimulus power.

\section{Results}

We investigated optically recorded maps for orientated grating stimuli and up to four different types of complex plaid stimuli in the central field representation of area 18 in 12 cats.

\section{Plaid tuning maps}

In order to assess whether area 18 population responses reflect only the orientation and direction of movement of the plaids' component gratings we have run tuning protocols with two different plaid stimuli, one strongly pattern-biased with black intersections (PB 1) and one depth-ordered non-pattern biased one (NPB 1, for details of stimulus construction see Materials and methods and Table 1), moving in eight different directions. Overall, plaid evoked activation was on average 
A

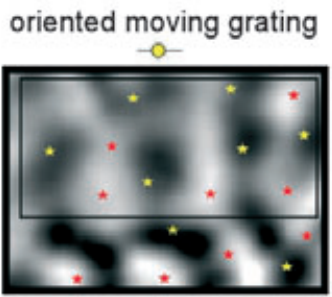

C
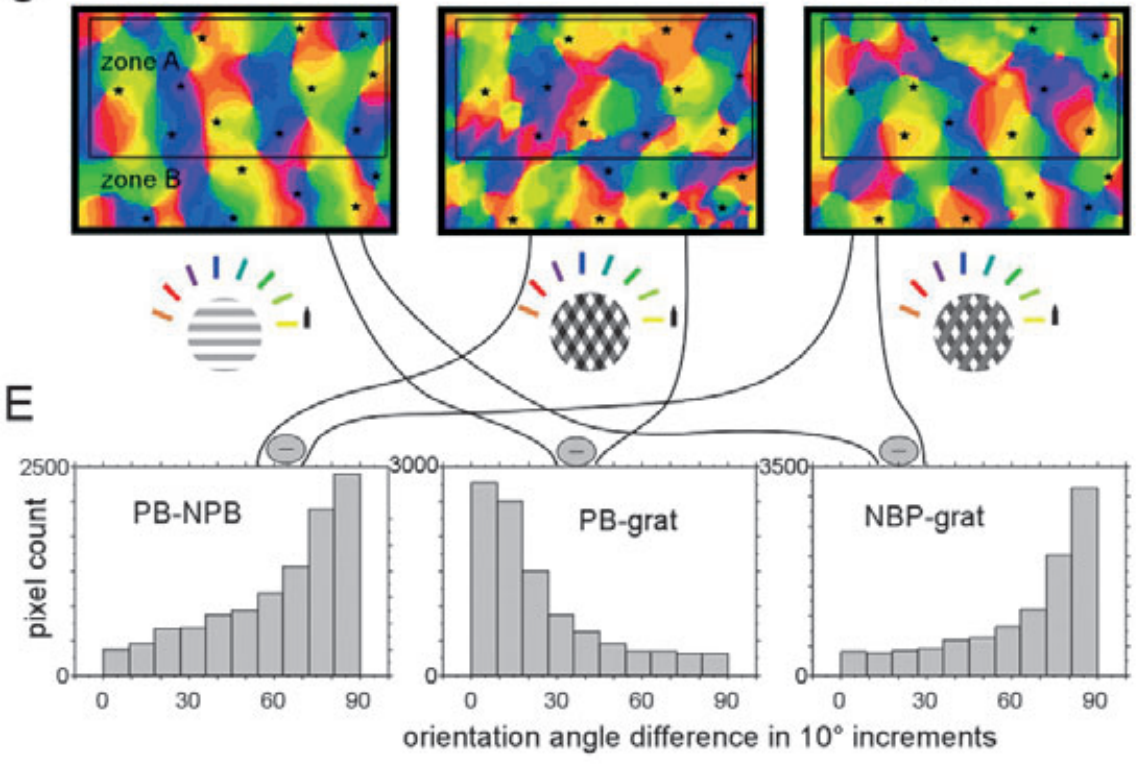
pattern-biased plaid
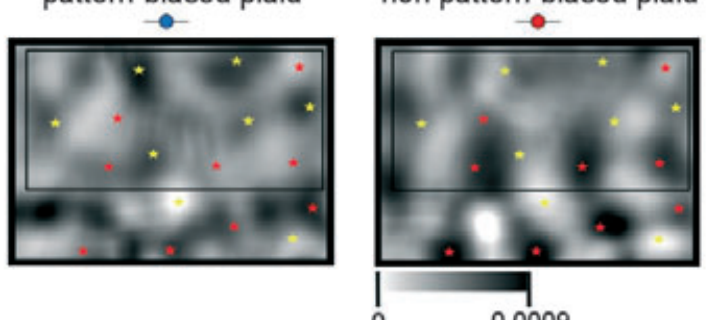

0

0.0009
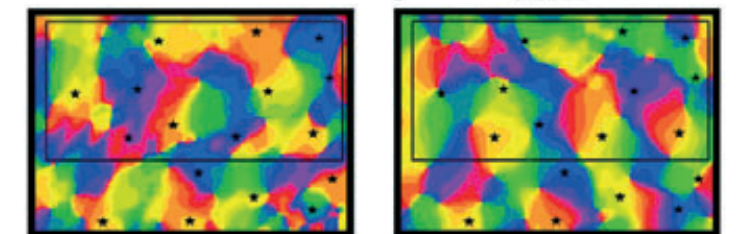

non pattern-biased plaid

$\mathrm{D}$
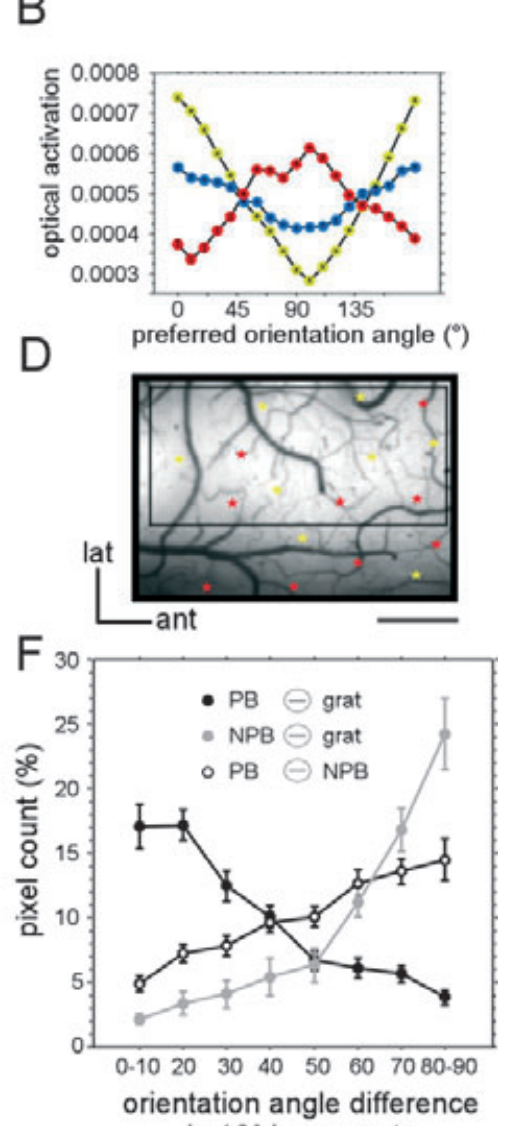

in $10^{\circ}$ increments

FIG. 2. Plaid tuning maps. Activity maps obtained from the same cortical site using gratings (left), a pattern-biased (PB 1, middle) and a non-pattern-biased plaid stimulus (NPB 1, right) moving into eight different directions (for details on stimulus construction, see Materials and methods). Maps are cocktail-blank normalized, no model was applied. Asterisks mark identical points in all maps. Single condition maps (A) and orientation response profiles (B) obtained for a horizontal grating $\left(0^{\circ}\right)$ moving upwards and two plaids moving in the upward pattern direction $\left(90^{\circ}\right)$. (A) Within the rectangular region of interest (zone A), the pattern-biased plaid (PB 1) evokes considerable activity in similar domains as the horizontal grating (yellow asterisks), whereas the non-pattern-biased plaid (NBP 1) quite strongly stimulates orthogonal domains (red asterisks). (B) Horizontal-vertical differential activity for the three types of stimuli in A plotted against the angles of the grating angle preference map. Both grating and PB 1 response profiles peak around $0^{\circ}$, the NPB 1 profile peaks around $90^{\circ}$. In addition, the $105^{\circ}$ orientation component of NPB 1 seems to evoke slightly stronger responses than the $75^{\circ}$ orientation component as indicated by a small satellite peak of the response profile in Fig. $1 \mathrm{~A}$. (C) Angle preference maps were computed by pixel-wise vector summation. The colour code (below the coloured maps) in all maps refers to the pattern direction/orientation (as in conventional angle maps opposite directions are summed) of motion of the plaid stimuli. Inside zone A, the map obtained by the patternbiased plaid (PB 1) is similar to the map obtained by the gratings and orthogonal to the map obtained by the non-pattern-biased plaid (NBP 1). Interestingly, outside the region of interest (peripheral zone B in A and B), the pattern-biased plaid map was orthogonal to the grating map and similar to the non-pattern-biased plaid map. (D) Vessel image of the recorded region. (E) Mean angular differences per pixel in $10^{\circ}$ steps between the different maps in the zone A. Note that the angular difference between maps obtained by pattern-biased plaids to grating maps was predominantly $0^{\circ}$, whereas it was predominantly at $90^{\circ}$ when comparing to the nonpattern-plaid map. (F) Average of the angular difference per pixel in $10^{\circ}$ steps between grating, pattern-biased and non-pattern-biased plaid maps from seven data sets (five cats). Grat, grating; NPB, non-pattern-biased; PB, pattern-biased. Cortical coordinates: lat, lateral; ant, anterior. Scale bars, $1 \mathrm{~mm}$.

approximately $25 \%$ lower than grating induced activation. Surprisingly, activations evoked by a pattern-biased plaid peaked in similar domains as those evoked by a grating stimulus moving into the pattern direction of motion in a large part of the recorded region (Fig. 2A, second column and Fig. 2B). Accordingly, the orientation angle map computed from stimulation with the plaid PB 1 closely resembled the orientation angle map obtained by classical grating stimulation in the pattern direction of motion (Fig. 2C, first and second column, Fig. 2E, second column). Thus, a strongly pattern-biased stimulus evoked the strongest activation in the domains activated by the pattern direction of motion and not in the domains activated by the plaids' component motion. In contrast to this and despite large physical similarities of both plaid stimuli the non-pattern-biased stimulus (NBP 1) best activated the complementary domains with orthogonal orientation and direction preference to those observed by pattern-biased stimulation
(Fig. 2A and C, third column; Fig. 2E, first column). If the pattern direction is $90^{\circ}$, the $0^{\circ}$-orientation domain is activated by the pattern model and the pattern-biased plaid. The respective components move into the $15^{\circ}$ and $165^{\circ}$ direction and thus should activate orientation domains of $75^{\circ}$ and $105^{\circ}$. As the $90^{\circ}$ orientation domain is closest to both of them it is jointly activated by non-pattern biased plaids $\left(90^{\circ}\right.$ from the orientation domain activated by pattern motion, see Fig. 2E, third column).

On average in five cats, the match between stimulation with nonpattern biased plaid 1 and grating stimulation of the 'in-between' component domains was larger than the match between pattern-biased plaid 1 stimulation and direct grating stimulation of pattern domains (Fig. 2F). In the peripheral part of the area 18 map, component-motion related activation seemed to predominate for both plaid stimuli, as angle maps resulting from both pattern-biased and non-pattern-biased 
plaid stimulation differed by $90^{\circ}$ from the grating map, and resembled each other (see zone B in Fig. 2C).

\section{Pattern motion activity in area 18}

In order to obtain a detailed view of the balance between pattern and component motion in area 18, stimulation with four plaid types with different degrees of pattern-bias was compared to stimulation with a grating moving at the same velocity and into the same direction as the vector of plaid pattern motion (pattern model) or to the sum of the activations obtained by stimulation with the plaids' individual component gratings (component model; Fig. 3). Our prediction was that only pattern-biased stimuli would evoke activity maps that strongly correlated with the pattern model. Furthermore, all stimuli should show correlations with the component model, which is explained by neural processing of local component contours that are common to all stimuli. The relative accordance with each of the model maps should be dependent on the relative bias for local or global processing (Stoner et al., 1990); for depth-ordered stimuli pattern bias should be minimal. Our results tightly match these predictions.

Plaid maps were calculated as differential maps by dividing maps obtained with plaid stimuli by the sum of maps obtained by the two component stimuli ('component divisor', CD) or by the map obtained with the grating moving in the pattern direction ('pattern divisor', PD). These procedures served to normalize plaid maps and to extract activation that was either not explained by the component grating stimulation or by the grating stimulation modelling the pattern direction of motion (for control procedures that did not use normalization to pattern and component divisors, see Materials and methods section). When divided by the $\mathrm{CD}$, maps evoked by different plaid stimuli revealed very different modulations in central area 18 (Fig. 3A). In most of the cases, plaid PB 1 (black intersections) activated similar domains as the grating moving into the pattern direction (pattern model). This was, to a smaller extent, also observed for the plaid PB 2 (white intersections). Thus, pattern-like modulations were evoked by pattern-biased plaids but sometimes also by non-pattern-biased plaids (NPB 2). This suggests that population signatures of pattern representations were detectable provided that stimuli exhibited clear-cut local intersections that introduced considerable bias for pattern motion. Maps evoked by the depth-ordered transparent plaid, which had no clear-cut intersections and thus the strongest bias towards component motion, were almost always rather flat when analysed for pattern motion activity (CD normalized, see also Fig. 4B).

However, all plaid maps revealed considerable component motion activity. When eliminating modulations explained by the PD (Fig. 3B), activation remained in regions topographically corresponding to the orientation domains activated by the single component gratings and the orientation domains between the two plaid components (vertical orientation domains). This shows that activity maps in area 18 can signal both pattern and component motion; as expected, component motion signals are present for all stimuli and additional pattern motion signals are only present if the stimulus corresponds to pattern motion.

When plaid stimuli were presented in the more peripheral parts of the visual field, e.g. in the most anterior part of area 18 on the surface of the lateral gyrus (more than approximately $-5^{\circ}$ of elevation), none of the plaid stimuli evoked pattern motion plaid activity and all maps resembled those obtained by the depth-ordered transparent plaid (data not shown).

The nature of the pattern and component motion activations became particularly evident when superimposing the normalized plaid maps onto the orientation preference map obtained by grating stimulation (Fig. 4A). In the colour-brightness coded CD normalized maps (revealing pattern-like activation), the two pattern-biased plaid stimuli (plaids with black or white intersections) activated regions centred on the orientation domains (yellow) that were also activated by the horizontally orientated and upward moving pattern model grating (Fig. 4B). This did not hold true for the non-pattern-biased plaid stimuli. However, on all colour-brightness coded PD normalized maps (revealing component-like activation) activation can be observed in regions corresponding to the orientation domains activated by the two single component gratings (red and green) and especially in domains representing the vertical orientation in between (blue).

To quantify these modulations, plaid specific activation after normalization with the respective divisor was plotted against the preference angle in the grating map (opposite directions in the gratings maps were lumped together, Fig. 5A). Quantification of the data set shown in Figs 3 and 4 clearly demonstrates that CD normalized maps (Fig. 5A, upper row) exhibited highest activity levels around the orientation/direction of the pattern model grating $\left(90^{\circ}\right)$ for patternbiased plaid 1, followed by pattern-biased plaid 2. Neither of the nonpattern-biased plaids showed any pattern-like activation in this example. For PD normalized maps (Fig. 5A, lower row), activity was observed mainly in the angle compartments, which are jointly activated by the sum of the single components (around $0 / 180^{\circ}$ ). This is representative of most of the data sets obtained in area 18 using this paradigm as becomes obvious in the grand average ( $n=33$, Fig. $5 \mathrm{~B})$. In order to compare different data sets from different cats, the anglespecific activation was expressed as per cent of the mean activation $(100 \%)$ of the respective normalized plaid map. Indeed, after CD normalization, this average analysis revealed the highest activity in the pattern-like orientation/direction domains $\left(90^{\circ}\right)$ for the pattern-biased plaid 1, followed by the pattern-biased plaid 2 and the non-patternbiased plaid 2. Only the depth-ordered stimulus (NBP 1), which is least consistent with the pattern model, did not evoke a peak at pattern direction as indicated by a dip in the average activation around $90^{\circ}$. When normalizing to the PD, for all plaid stimuli, strong activity remained in the domains responding to the local orientation and direction of the components, or to both of them $\left(0^{\circ} / 180^{\circ}\right)$. This type of activity was lowest for the pattern-biased plaid 1 with black intersections, which reveals the highest pattern-bias and the highest pattern-like activity. For statistical evaluation we separated activity distributions into two angle compartment bins of equal size (compartment $\mathrm{P}, 45-134^{\circ}$ and compartment $\mathrm{C}, 135-44^{\circ}$ ) and tested them against each other using a Mann-Whitney $U$-test. For CD normalized maps, activity around $90^{\circ}$, the pattern orientation/direction of motion (compartment $\mathrm{P}$ ), was significantly higher than around $0^{\circ}$ (compartment $\mathrm{C}$ ) only for plaid PB $1(P<0.002)$. For pattern corrected maps, activity in compartment $\mathrm{C}$ was significantly higher than around $90^{\circ}$ in all cases $(P<0.002)$. Again, this suggests that a significant common component-like response is present in all maps, unlike the pattern-like response.

The Spearman rank correlation between the plaid maps and the model maps was computed to obtain a graded picture of pattern- and component-like activity independent of the reference angle map (Fig. 6A and B). Respective correlation coefficients were labelled pattern and component indices, respectively. This analysis revealed the highest pattern indices for the pattern-biased plaid 1, followed by the pattern-biased plaid 2 and very low indices for both non-pattern biased plaids. However, component indices were high for both nonpattern-biased plaids and also pattern-biased plaid 2. Component indices were only low for pattern-biased plaid 1 (the stimulus with the highest pattern-bias). Plotting the individual indices (Fig. 6A) also 
A

PB 1
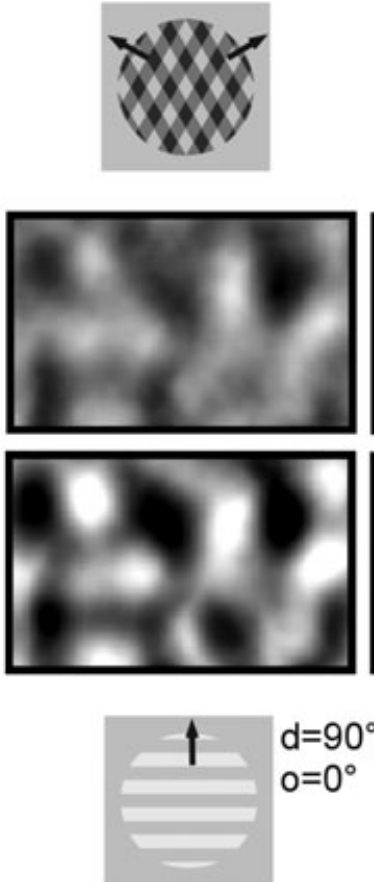

B
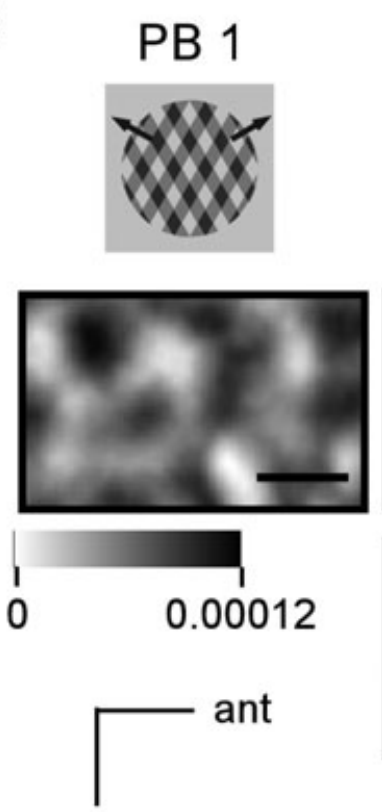

lat
PB 2
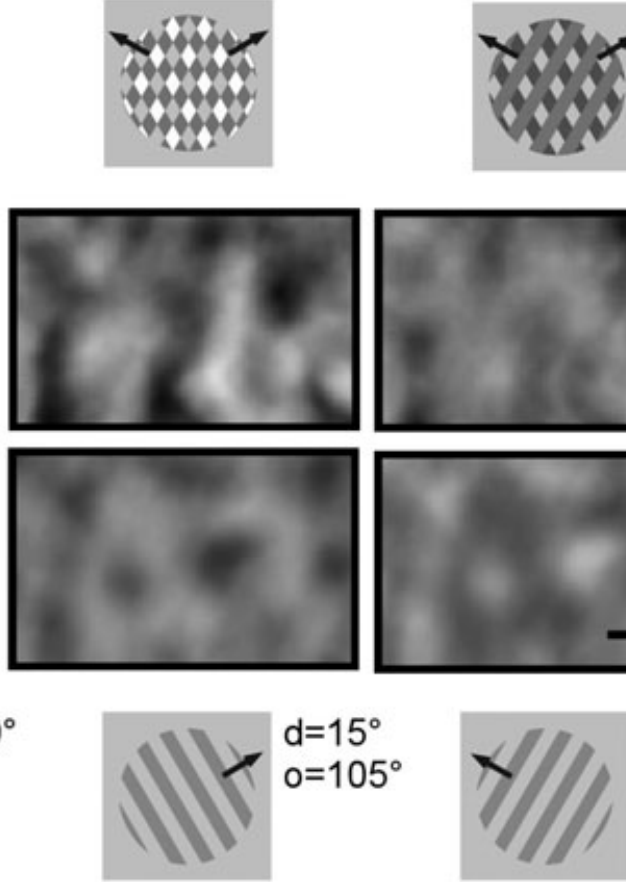

$d=165^{\circ}$

$0=75^{\circ}$
NPB 2
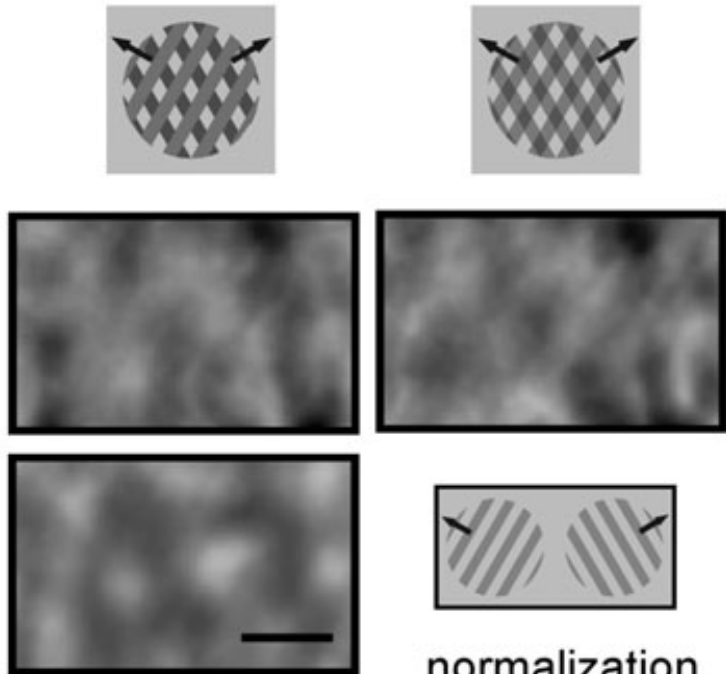

factor

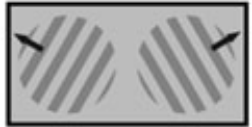

\section{normalization}

factor
NPB 2

NPB 1
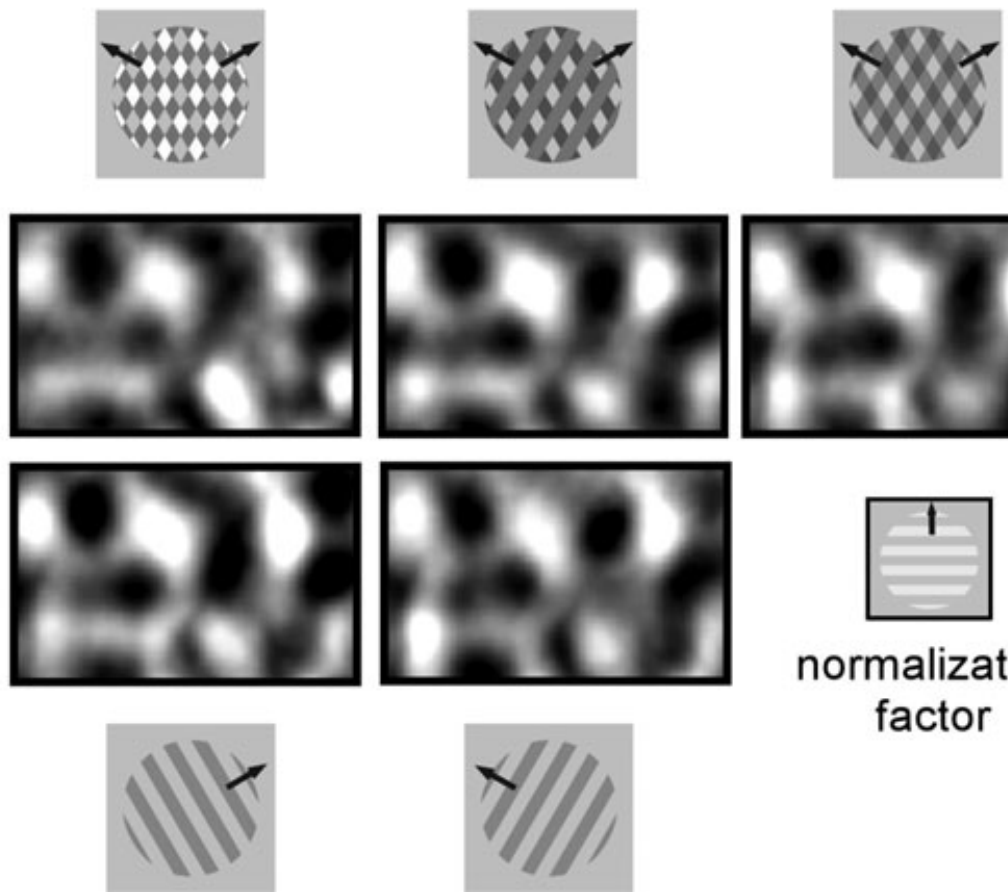
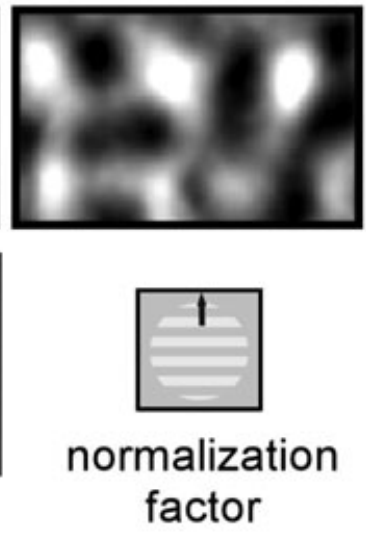

FIG. 3. Differential activity maps evoked by plaid stimuli. (A) Differential plaid activity and model maps. All maps are divided by the sum of the maps evoked by the two components ('normalization factor', CD, stimuli in right lower inset). Dark domains correspond to pattern motion evoked activity, i.e. activity not explained by the component model. Strong modulations are evoked by the pattern-biased plaids. Note that these modulations resemble those evoked by the pattern model (horizontal grating moving upwards). (B) Differential plaid activity maps divided by the map evoked by the pattern model grating ('normalization factor', PD, stimulus in right lower inset). Dark domains correspond to component motion evoked activity, i.e. activity not explained by the pattern model. Results are quite complementary to the ones observed in A; activation is weaker with the pattern-biased plaid (PB 1) that exhibited stronger activity in A than with the non-patternbiased conditions (NPB 1 and 2). Note that all normalized maps evoked by plaids resemble closely those evoked by the single component gratings, which shows that a common denominator of local component processing is always present. d, direction of movement; o, orientation. Other abbreviations as in Fig. 2 . Scale bars, $1 \mathrm{~mm}$. 
A

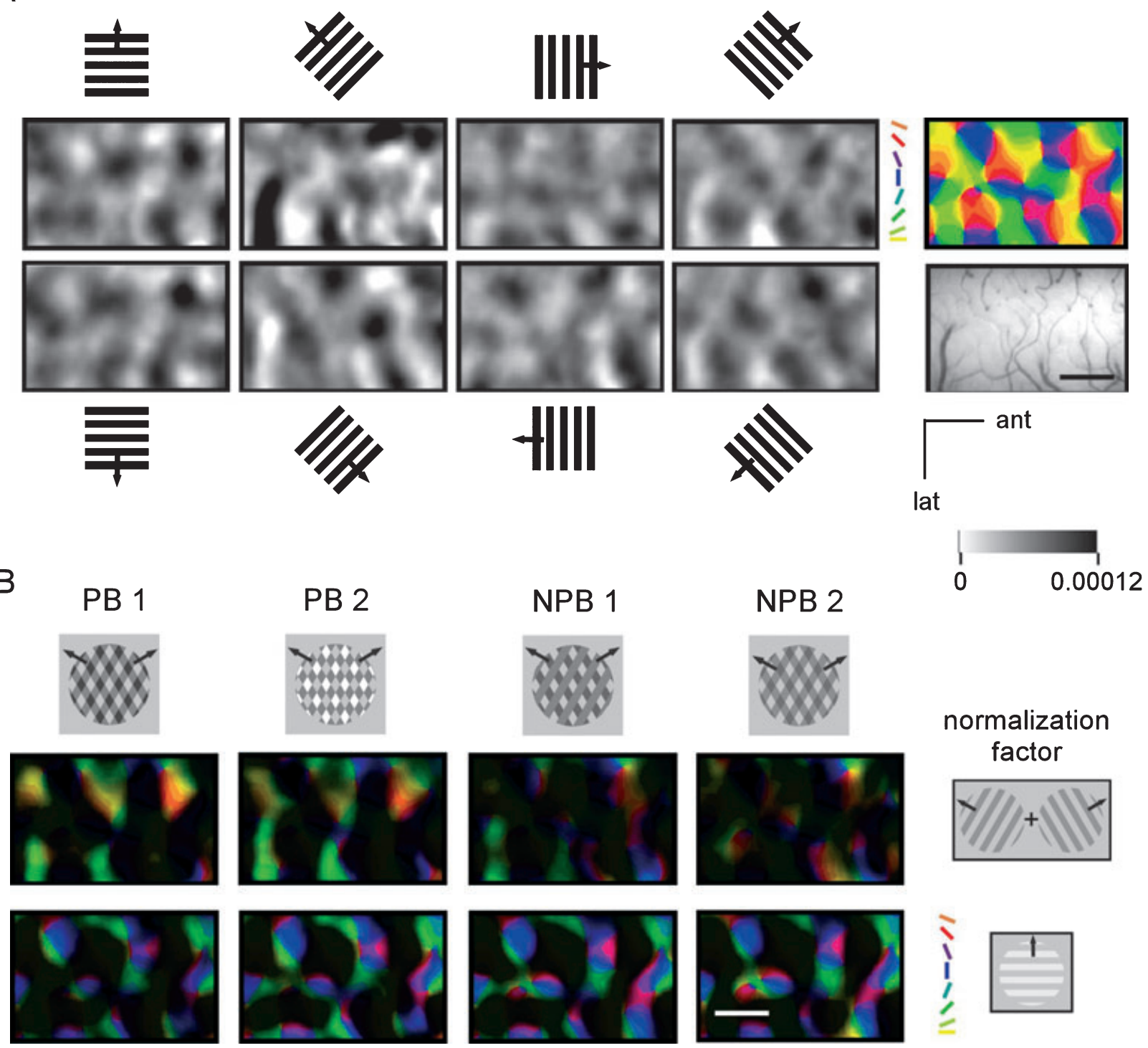

FIG. 4. Pattern- and component-plaid activity superimposed on the orientation map. (A) Single orientation maps in area 18 evoked by stimulation with squarewave gratings moving in eight different directions at $4 \mathrm{deg} / \mathrm{s}$ and $0.5 \mathrm{cycle} / \mathrm{deg}$ as indicated by the sketches above or below each activity map. Right panels, upper: conventional angle map computed by pixelwise vectorial addition of the single conditions. The preferred angle of orientation is colour-coded according to the scheme on the left. Lower: video image of the recorded cortical region. (B) Plaid activity maps superimposed on the orientation preference map shown in A. Plaid activity level is coded by the brightness of colours; bright colours indicate strong activation by plaids; colour-code as in A. Right insets: stimuli used for normalization. The upper row shows CD normalized maps, thus revealing activity evoked by pattern motion (data taken from Fig. 3A), and the lower row PD normalized maps, thereby highlighting activity evoked by component motion (data from Fig. 3B). Note that the pattern-biased plaids evoke stronger activity in the yellow orientation domains driven by horizontal contours than the other plaid conditions (upper row) when normalized to the CD. All PD normalized plaid stimuli (showing activity evoked by the component-model, lower row) evoked activation in the oblique orientation domains (green and pink colours) corresponding to the plaids' components and in the intermediate vertical orientation domains (blue) that are probably partly activated by both components. Conventions as in Fig. 2. Scale bars, $1 \mathrm{~mm}$.

demonstrated that pattern and component correlation could vary for different plaids over a wide range. In individual data sets, high pattern indices for non-pattern biased plaids as well as high component indices for pattern-biased plaids occurred occasionally.

In theories on visual perception, it is common to describe processing in the first stages of the visual system as a sequence of filtering processes specialized to analyse the spatial pattern of light in the retinal image according to its spatial frequency components. Given that all the possible combinations of spatial frequency and orientation tuning are represented in the response properties of striate cortical neurons in a modular manner the primary visual cortex has been also discussed to be composed of overlapping tissue units performing a segment-wise Fourier analysis of the retinal image (e.g. De Valois \& De Valois, 1990). In order to evaluate the possible contribution of spatiotemporal Fourier energy of our stimuli to our maps' pattern and component indices Fourier components were computed for all four plaid stimuli (Fig. 7A; according to Stoner \& Albright, 1996). It turned out that the plaid stimulus exhibiting the highest relative Fourier power in the two component directions (NPB 1) and the lowest in the pattern direction was also the one with the highest component index and lowest pattern index (Fig. 7B). In general, the averages of pattern/component indices and Fourier power followed the same 


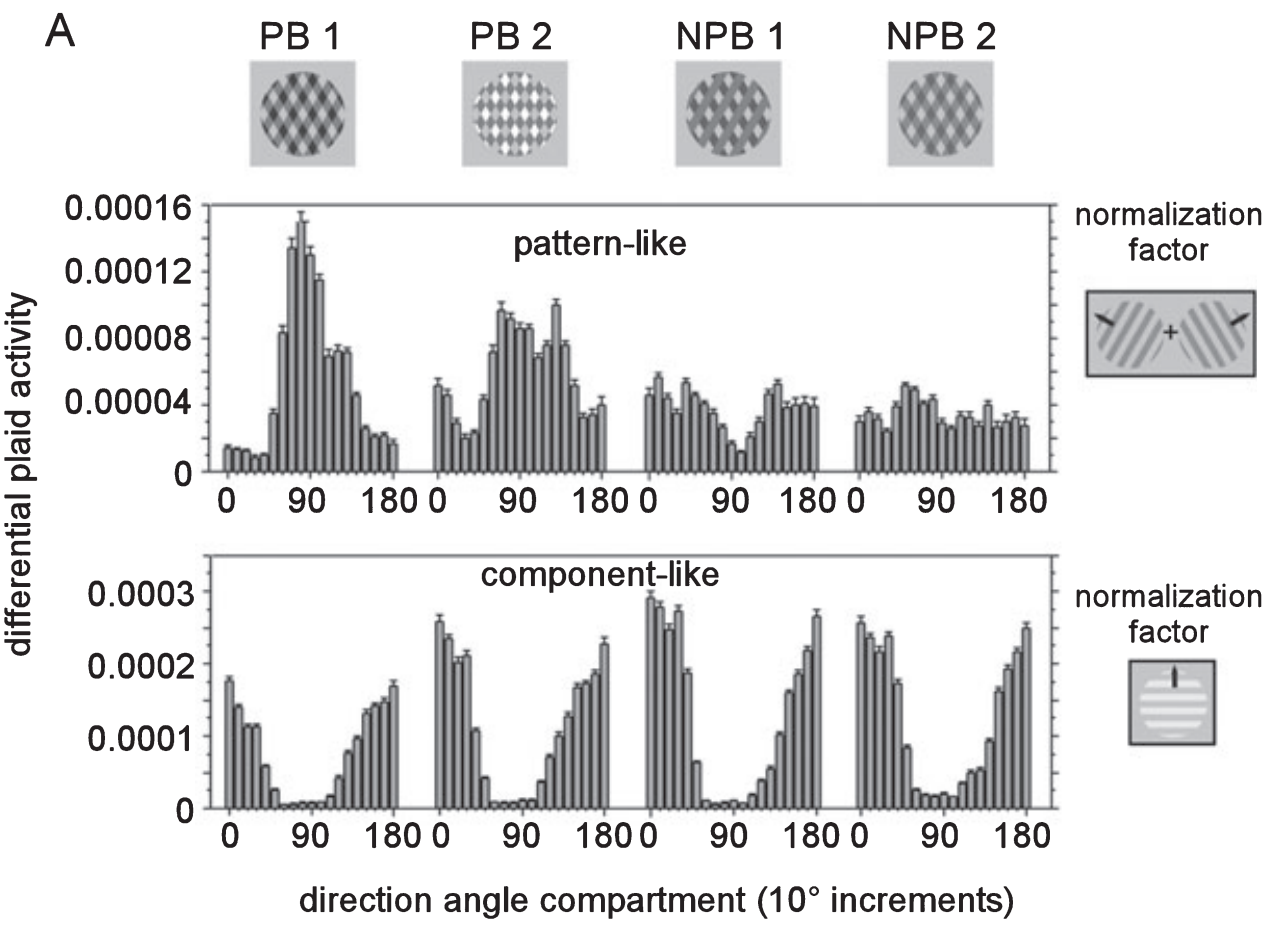

B

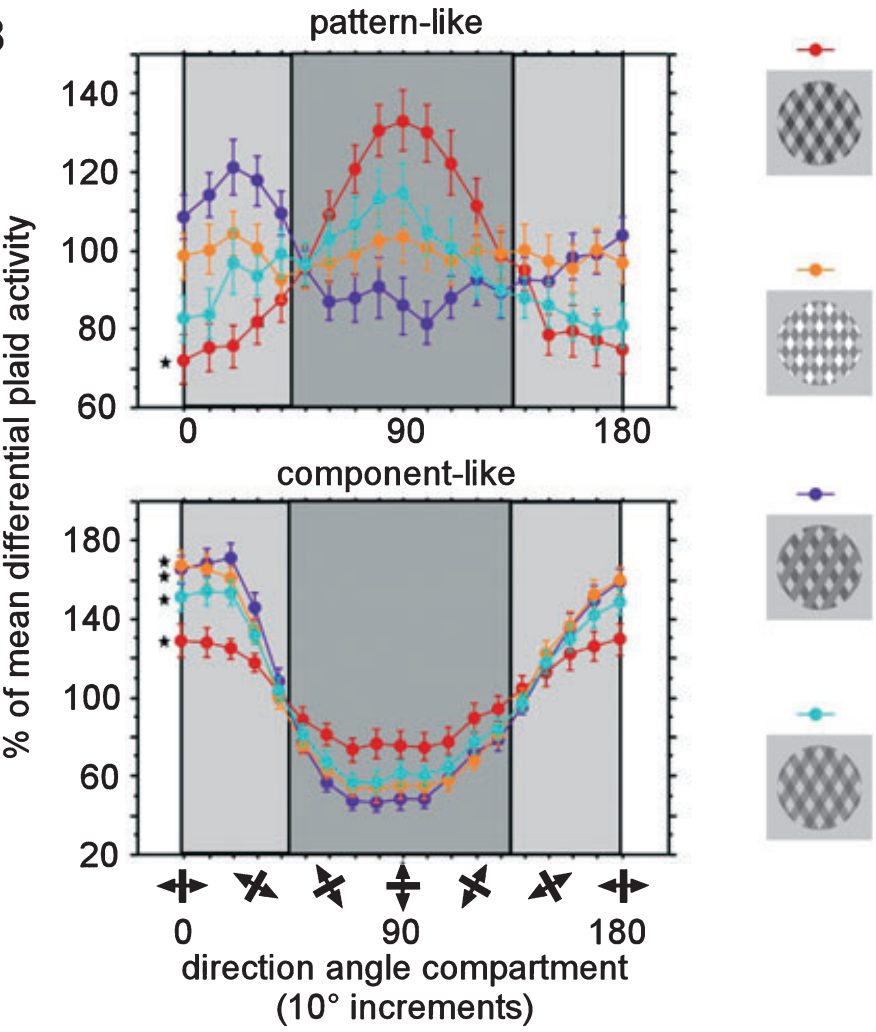

FIG. 5. Relative pattern- and component-like activity in individual orientation preference compartments. (A) Quantification of the data set illustrated in Fig. 4. Right insets: stimuli used for normalization. Activity (as illustrated by colour saturation in Fig. 4) evoked by different plaid stimuli within different angle compartments of the grating map. Upper row: CD normalized activity (yielding pattern-motion evoked activity), lower row: PD normalized activity (yielding component-motion evoked activity). Opposite directions of movement are lumped together in orientation domains. Angular components are binned in 10-degree steps. Labels indicate the angle of direction of motion. All stimuli evoked component-motion related activity in vertical orientation domains, but only pattern-biased stimuli evoked pattern-motion related activity horizontal orientation domains. (B) Relative CD and PD normalized activity $( \pm$ SEM) of all data sets $(n=33)$ from 11 cats in percentage of the mean activity per plaid evoked and normalized map within different angle compartments. Asterisks indicate significant (Mann-Whitney$\mathrm{U}, P<0.002$ ) activity differences in the two angle compartment bins of equal size (dark grey for the pattern direction of motion: $45-134^{\circ}$, light grey for the component direction of motion: $135-44^{\circ}$ ). On average, plaid PB 1 (black intersections) was the only plaid evoking significant activity not explained by the component model. However, the only plaid clearly not evoking a peak at pattern direction was the one with absent grating intersections and thus least consistent with the pattern model (NPB 1, the depth-ordered stimulus). All plaids evoked strong activity explained by the component model. 

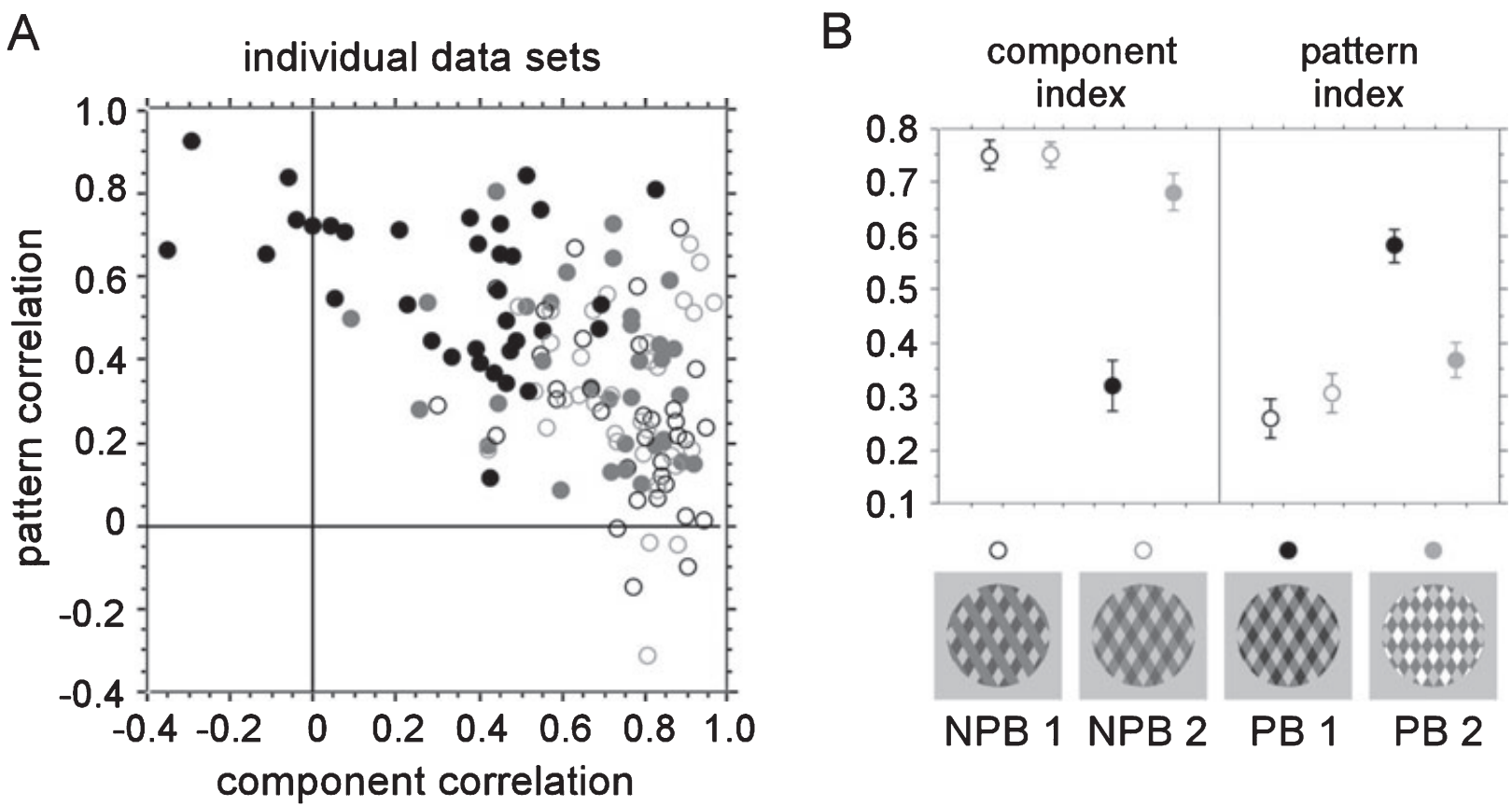

FIG. 6. Map pattern and component correlation indices. (A and B) Map pattern and component correlation indices (for details see Materials and methods) obtained with the different plaid stimuli (according to the symbols on the right). Spearman rank correlation coefficients were computed pixel-wise in a large region-of-interest between single plaids evoked maps and the map evoked by the pattern or the component model gratings. Here, all maps have been normalized to a cocktail blank of all seven stimulus conditions before computing the correlation. (A) Pattern correlation coefficients are plotted against component model coefficients for all 33 single data sets. Note that the pattern-biased plaid with dark intersections (PB1, filled black circles) induced higher pattern than component correlations. Vice versa the stimuli least consistent with pattern motion (non-pattern-biased and/or depth-ordered, open circles) evoked higher component than pattern correlations. The stimulus with white intersections seems to contain a similar amount of pattern and component-motion related information. (B) Component and pattern indices computed by averaging the correlation values per stimulus type. The highest component index is evoked by the depth-ordered transparent plaid (NPB 1), followed by a sequence in which the pattern-biased plaid with dark intersections (PB 1) has the lowest component index. For the pattern index, the sequence is reversed.

trend. However, plaid NPB 1 and 2 have similar average component indices (NPB $10.75 \pm 0.027$; NPB $20.75 \pm 0.022$ ) but Fourier power in the component directions differs by more than $15 \%$. Secondly, pattern indices cover the same range as component indices although stimulus power in pattern direction is orders of magnitude smaller $(0.1-12 \%)$ than in component direction $(20-97 \%)$.

Further, a rank correlation revealed that only a part of the large variance in the optical imaging data could be explained by the Fourier spectra of the different plaid stimuli (Kendall rank correlation, component direction: tau $=0.36$; pattern direction: tau $=0.32$ ). This large variance results from the fact that the distribution of pattern/component indices in single data sets does not follow the trend predicted by the Fourier power spectra. In the data set illustrated in Figs 3, 4 and 5A, we see a pattern index of 0.805 and a component index of 0.825 for the PB 1 stimulus, a pattern index of 0.379 and a component index of 0.92 for the NPB 1 stimulus (see arrows in Fig. 7B).

Thus, we concluded that Fourier energy content of the stimuli is to some extent mirrored in the average response of all data sets but cannot fully account for our findings.

To further exclude the possibility that our results were based on local contrast changes we performed a control experiment in which we changed the ratio between the width of bars and background in the gratings of plaid PB 1 (duty cycle; Fig. 7C). This is known to reverse figure-ground assignment because non-transparent grating intersections change to background and the lighter background changes to intersections. Thus, in the second half of the duty cycle, this leads to a perceptual switch from pattern to component motion (see Stoner \& Albright, 1996). In agreement with this prediction the pattern index in the activity maps drops from values observed for pattern-bias $(0.7$ at duty cycle 0.2$)$ to non-pattern bias $(0.31$ at duty cycle 0.8$)$.

\section{Discussion}

The main results of the present study indicate that complex 2D patternmotion representations are detectable at the population level already in early visual areas. We have recorded intrinsic optical signals from area 18 while stimulating with different plaid stimuli and we analysed the amount of component- and pattern-like evoked activation in the neural representation of these stimuli. Most interestingly and quite unexpected given the electrophysiological literature, plaid stimuli with a pattern-bias evoked responses matching the location of pattern model maxima in area 18 orientation maps. All plaid stimuli also correlated to a variable degree with a model of component motion, which is based on neural processing of the local contours that are common to all plaid stimuli. Confirming our predictions, the relative correlation with each of the two models was dependent on the relative bias of the respective stimulus for component or pattern motion (Stoner et al., 1990).

The pattern-biased stimulus (PB 1) that evoked the strongest and significant correlation with the pattern model in the majority of data sets was also the stimulus that evoked the lowest correlation with the component model. Activity maps obtained with depth-ordered stimuli almost always correlated better with the component model than any of the activity maps obtained with all other stimuli, but exhibited the lowest correlation with the pattern model. This observation is likely to be explained with the notion that depth-ordered stimuli are strongly biased for 1D contour processing. Intermediate sets (PB 2 
A

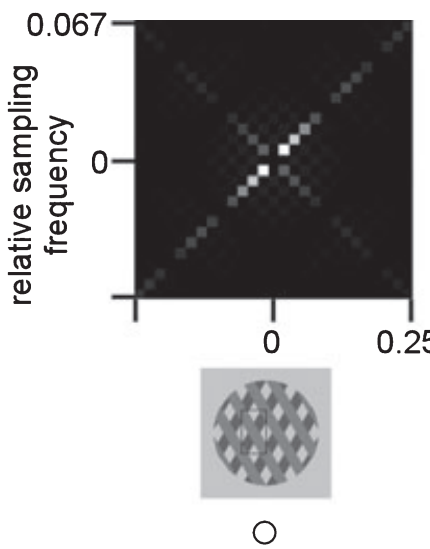

B

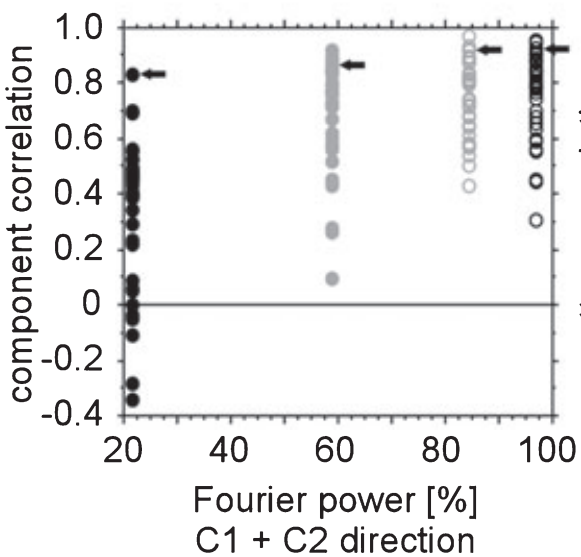

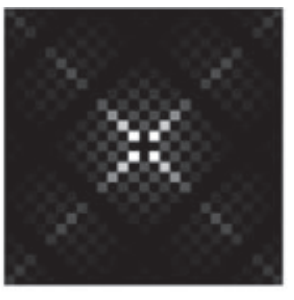
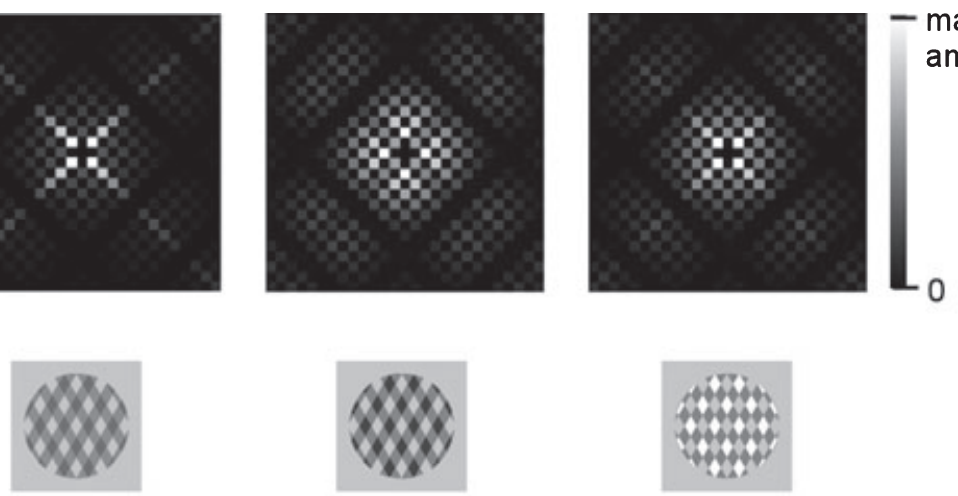

○

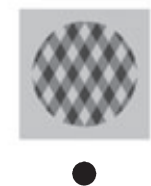

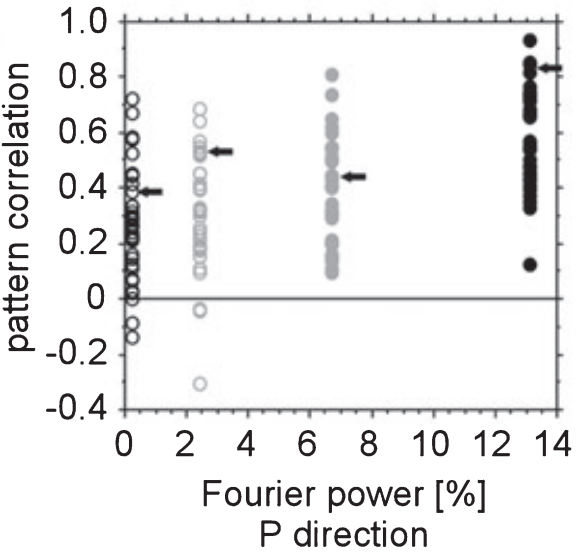

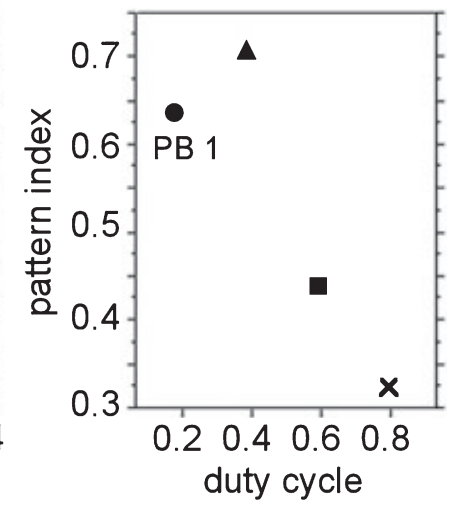

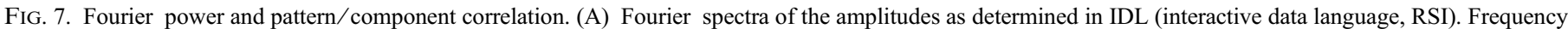

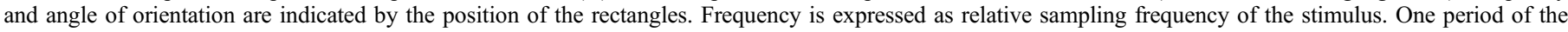

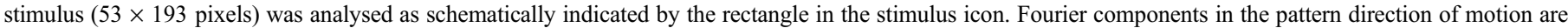

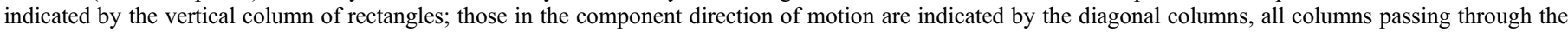

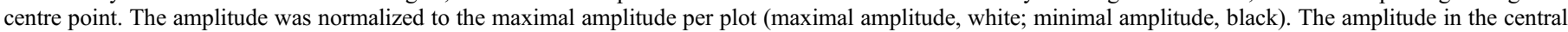

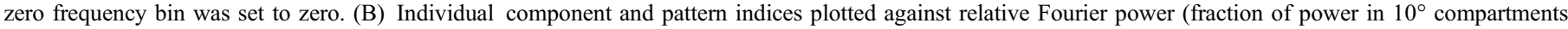

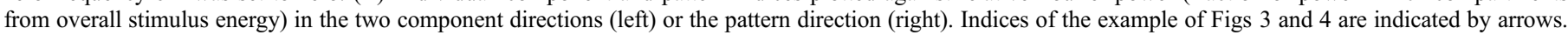

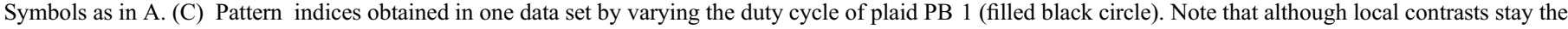

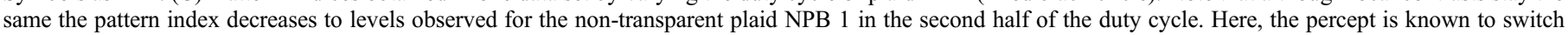
from pattern to component motion (Stoner et al., 1996).

and NPB 1) showed correlations of variable strength with both models.

Although the relative component- and pattern-like modulations follow the predictions made by the degree of pattern bias, all stimuli revealed significant component-like modulations. This is to be expected because all plaid stimuli contain local 1D-orientated elements that are likely to activate domains preferring the orientation and direction of motion of the plaids' components.

Recently, optically recorded maps in ferret visual cortex to textures moving into directions other than that perpendicular to their orientation also activated orientation domains not corresponding to the respective stimulus' orientation (Basole et al., 2003). These results have been suggested to be explained by a 3D energy space model of a combination of linear filters in V1 (Baker \& Issa, 2005; Mante \& Carandini, 2005). According to this model, plaid evoked maps can be interpreted as population activity in a $3 \mathrm{D}$ space predicted from the plaid stimulus energy. However, such a model is difficult to reconcile for our data for several reasons. First, for individual data sets pattern and component activity were not predictable by Fourier energy components assessed from the stimulus. For example, high pattern indices co-occurred with high component indices for the same stimulus. Second, shifts in spatiotemporal energy of the stimulus did not shift the type of representation evoked because component domains were always activated. Third, pattern and component correlation coefficients (Fig. 6) for the very same visual stimulus varied between different data sets in different cats indicating influences of cortical processing modes on the activity patterns that are independent of the actual visual input. Fourth, a stimulus where local contrasts did not change, and with pattern-bias constructed by only modifying the duty cycle, produced pattern and component correlations predictable from psychophysics. Thus, we assume that a static model based on stimulus energy including only V1 processing cannot fully account for our observations.

Along a similar line as the Fourier energy hypothesis goes an interpretation by Movshon et al. (2003). The authors argue that plaids stimuli constructed in a non-additive way always contain a third 
component and thus evoke the response of low level filters to stimulus components moving into pattern direction. Even though all of our stimuli contain a third added component, the plaid that is closest to a non-additive case (NPB 2) evokes much lower pattern motion than the two non-transparent cases PB 1 and PB 2.

While pattern-motion responses in early visual areas are barely observed in single and multiunit responses, particularly in anaesthetized animals (e.g. Movshon et al., 1985; Gizzi et al., 1990; CasteloBranco et al., 2000), it is surprising that they can be detected in metabolic population responses. One reason might be the choice of different stimulus parameters (Movshon et al., 2003). Another reason for this discrepancy could be that earlier electrophysiological studies focused on investigations of multiunit responses. These could obscure single pattern-selective neurons and thus, the actual number of patternselective neurons in V1 would be underestimated. However, also single-unit recordings in primate V1 revealed only limited pattern selectivity (Guo et al., 2004). Thus, there might be an explanation for our observations other than the different sampling basis and scope in optical imaging. Recordings of single and multiunit responses measure only action potentials that do not show subthreshold synaptic processes, which are thought to substantially contribute to the responses captured by optical imaging (Das \& Gilbert, 1995; Toth et al., 1996; Niessing et al., 2005) and other measurements of cerebral blood flow and oxygenation (Mathiesen et al., 1998; Logothetis et al., 2001). There is recent evidence that brain imaging techniques reveal synaptic processes hardly reflected in spiking activity (Logothetis et al., 2001; Schmidt et al., 2004; Niessing et al., 2005) which might contribute also to our finding.

Two models have been suggested to account for the perceived coherence of plaid motion (Adelson \& Movshon, 1982). One model proposes that the perceived motion is determined by the motion of the intersections ('blobs') and that motion is particularly biased towards the true plaid direction of motion through a low level 'blob tracking mechanism' (Alais et al., 1994). Our results are consistent with such a mechanism as pattern responses were particularly strong for plaids with high intersection contrast. As neurons in early visual areas are very contrast-sensitive, high intersection contrast may increase the saliency of the blobs in a feature tracking mechanism and, thus, serve to bias activity patterns towards a reflection of pattern motion. However, contrast sensitivity is even higher in peripheral parts of the visual field where the even larger receptive fields could integrate over more 'blobs'. This contrasts with our observation that in peripheral retinotopic regions there was only a representation of component properties. Moreover, we found a decrease in the pattern index when we increased the duty cycle and thereby enhanced the compatibility of the stimulus with transparency and the percept of component motion without changing local contrasts. Therefore, we conclude that more important than contrast was whether stimuli were constructed such that $1 \mathrm{D}$ processing is biased against 2D processing, such as it occurs in depth-ordered plaids, or not. Taken together, these arguments do rather favour a 2D contour-tracking mechanism to underlie pattern motion maps in area 18. Therefore, we hypothesize that the pattern motion cells in early areas respond more strongly than component cells to corners and intersections because these are $2 \mathrm{D}$ stimuli and that the occurrence of pattern motion-like activity does not depend on a blob-tracking mechanism.

The second model for the perceived coherence of plaid motion assumes that the true direction of motion of a plaid pattern is determined by the intersection of constraints rule, which defines a vector in a two-dimensional velocity space where direction and velocity of the plaid's components intersect. The intersection of constraints mechanism is thought to require two successive stages of processing. The first stage evaluates the directions of movements of the local contours and neurons in the second stage compute the true direction of motion of the composite object by integrating over the output of the local analysers. Neurons belonging to the second stage have rarely been found in early visual areas, which might be attributed to their receptive field size not allowing for integration over large enough parts of the composite object. Thus, our finding of ample pattern motion-like activity in area 18 is rather surprising. However, it could fit into the two-stage model if one considers feedback from higher visual areas where neurons have larger receptive fields and can contribute to the second stage processing level. Therefore, it is possible that our optical recordings reflect input activity from back projections that remain undetected by multior single-unit recordings measuring only the neuronal output. Indeed, the variable correlations with both pattern- and component-models in our maps are consistent with variable correlations as a function of stimulus coherence described by Stoner and Albright for single neurons in MT (Stoner \& Albright, 1992). Here, stimulusdriven modulation was also more pattern-like when challenged with a non-transparent stimulus and the modulation becomes more component-like with the increase of physical and perceptual transparency in the stimulus. In conclusion, our findings suggest that central area 18 in the cat has access to pattern-motion signals at the population level. Given the rare occurrence of single pattern selective neurons in these areas indicates that area 18 neurons may not necessarily express this signature in their suprathreshold response. Future studies should address the role of intrinsic or feedback connections for the emergence of such representations in primary visual cortex.

\section{Acknowledgements}

We wish to thank Wolf Singer for his support and encouragement of this work. We are grateful to Sandra Schwegmann for excellent technical assistance, to Michael Stephan for help with analysis programs and to Will Barnes, Yvonne Egenolf, Michael Lutzenburg and Michael Niessing for assistance in some of the experiments. This work was supported by MPG, DAAD, and NSF.

\section{Abbreviations}

$\mathrm{CD}$, component divisor; $\mathrm{PB}$, pattern biased; PD, pattern divisor; NPB, nonpattern biased.

\section{References}

Adelson, E.H. \& Movshon, J.A. (1982) Phenomenal coherence of moving visual patterns. Nature, 300, 523-525.

Alais, D., Wenderoth, P. \& Burke, D. (1994) The contribution of onedimensional motion mechanisms to the perceived direction of drifting plaids and their after effects. Vis. Res., 34, 1823-1834.

Albright, T.D. (1984) Direction and orientation selectivity of neurons in visual area MT of the macaque. J. Neurophysiol., 52, 1106-1130.

Baker, T.I. \& Issa, N.P. (2005) Cortical maps of separable tuning properties predict population responses to complex visual stimuli. J. Neurophysiol., 94, 775-787.

Basole, A., White, L.E. \& Fitzpatrick, D. (2003) Mapping multiple features in the population responses of visual cortex. Nature, 423, 986-990.

Castelo-Branco, M., Goebel, R., Neuenschwander, S.T. \& Singer, W. (2000) Neural synchrony correlates with surface segregation rules. Nature, $\mathbf{4 0 5}$, 685-689.

Das, A. \& Gilbert, C.D. (1995) Long-range horizontal connections and their role in cortical reorganization revealed by optical recording of cat primary visual cortex. Nature, 375, 780-784. 
De Valois, R.L. \& De Valois, K.K. (1990) Spatial Vision. Oxford University Press, Oxford.

Galuske, R.A., Schmidt, K.E., Goebel, R., Lomber, S.G. \& Payne, B.R. (2002)

The role of feedback in shaping neural representations in cat visual cortex. Proc. Natl Acad. Sci. USA, 99, 17083-17088.

Gegenfurtner, K.R., Kiper, D.C. \& Levitt, J.B. (1997) Functional properties of neurons in macaque area V3. J. Neurophysiol., 77, 1906-1923.

Geisler, W.S., Albrecht, D.G., Crane, A.M. \& Stern, L. (2001) Motion direction signals in the primary visual cortex of cat and monkey. Vis. Neurosci., 18, 501-516.

Gizzi, M.S., Katz, E., Schumer, R.A. \& Movshon, J.A. (1990) Selectivity for orientation and direction of motion of single neuorns in cat striate and extrastriate visual cortex. J. Neurophysiol., 63, 1529-1543.

Guo, K., Benson, P.J. \& Blakemore, C. (2004) Pattern motion is present in V1 of awake but not anaesthetized monkeys. Eur. J. Neurosci., 19, 10551066.

Li, B., Chen, Y., Li, B.-W., Wang, L.-H. \& Diao, Y.-C. (2001) Pattern and component motion selectivity in cortical area PMLS of the cat. Eur. J. Neurosci., 14, 690-700.

Logothetis, N.K., Pauls, J., Augath, M., Trinath, T. \& Oeltermann, A. (2001) Neurophysiological investigation of the basis of the fMRI signal. Nature, 412, 150-157.

Mante, V. \& Carandini, M. (2005) Mapping of stimulus energy in primary visual cortex. J. Neurophysiol., 94, 788-798.

Marr, D. \& Ullman, S. (1981) Directional selectivity and its use in early visual processing. Proc. R. Soc. Lond. B Biol. Sci., 211, 151-180.

Mathiesen, C., Caesar, K., Akoren, N. \& Lauritzen, M. (1998) Modification of activity-dependent increases of cerebellar blood flow by excitatory synaptic activity and spikes in rat cerebellar cortex. J. Physiol., 512, 555-566.

Merabet, L., Desautels, A., Minville, K. \& Casanova, C. (1998) Motion integration in a thalamic visual nucleus. Nature, 396, 265-268.

Movshon, J.A., Adelson, E.A., Gizzi, M.S. \& Newsome, W.T. (1985) The analysis of moving visual patterns. In Chagas, C., Gattass, R. \& Gross, C.,
(Eds), Pattern Recognition Mechanisms. Vatican Press, Rome, pp. 117151.

Movshon, J.A., Albright, T.D., Stoner, G.R., Majaj, N.J. \& Smith, M.A. (2003) Cortical responses to visual motion in alert and anesthetized monkeys. Nature Neurosci., 6, 3.

Niessing, J., Ebisch, B., Schmidt, K.E., Niessing, M., Singer, W. \& Galuske, R.A.W. (2005) Hemodynamic signals correlate tightly with synchronized gamma oscillations. Science, 309, 948-951.

Pack, C.C., Berezovskii, V.K. \& Born, R.T. (2001) Dynamic properties of neurons in cortical area MT in alert and anaesthetized macaque monkeys. Nature, 414, 905-908.

Rodman, H.R. \& Albright, T.D. (1989) Single-unit analysis of pattern-motion selective properties in the middle temporal visual area (MT). Exp. Brain Res., 75, 53-64.

Scannell, J.W., Sengpiel, F., Tovee, M.J., Benson, P.J., Blakemore, C. \& Young, M.P. (1996) Visual motion processing in the anterior ectosylvian sulcus of the cat. J. Neurophysiol., 76, 895-907.

Schmidt, K.E., Singer, W. \& Galuske, R.A. (2004) Processing deficits in primary visual cortex of amblyopic cats. J. Neurophysiol., 91, 16611671.

Stoner, G.R. \& Albright, T.D. (1992) Neural correlates of perceptual motion coherence. Nature, 358, 412-414.

Stoner, G.R. \& Albright, T.D. (1996) The interpretation of visual motion: evidence for surface segmentation mechanisms. Vis. Res., 36, 1291-1310.

Stoner, G.R., Albright, T.D. \& Ramachandran, V.S. (1990) Transparency and coherence in human motion perception. Nature, 344, 153-155.

Thiele, A. \& Stoner, G.R. (2003) Neuronal synchrony does not correlate with motion coherence in cortical area MT. Nature, 421, 366-370.

Toth, L.J., Rao, S.C., Kim, D.-S., Somers, D. \& Sur, M. (1996) Subthreshold facilitation and suppression in primary visual cortex revealed by intrinsic signal imaging. Proc. Natl Acad. Sci. USA, 93, 9869-9874.

Wallach, H. (1935) Über visuell wahrgenommene Bewegungsrichtung. Psychologische Forsch, 20, 325-380. 